\title{
Letter to the Editor concerning "Particulate and non-particulate steroids in spinal epidurals: a systematic review and meta- analysis" by Feeley IH, Healy EF, Noel J, Kiely PJ, and Murphy TM (Eur Spine J; 2016): DOI 10.1007/s00586-016-4437-0
}

\author{
Harsha Shanthanna $^{1} \cdot$ Kamath Sriganesh $^{1,2}$
}

Received: 15 April 2016/Revised: 12 May 2016/Accepted: 12 May 2016/Published online: 19 May 2016

(c) Springer-Verlag Berlin Heidelberg 2016

We read the systematic review with great interest by Feeley et al. titled "Particulate and non-particulate steroids in spinal epidurals: a systematic review and meta-analysis" published in the recent issue of the journal [1]. The authors should be congratulated for this review on an important clinical topic. However, we wish to highlight some important limitations regarding this review.

The authors included only studies of transforaminal epidural steroid injections (TFESI) and excluded any studies of interlaminar epidural steroid injection (ILESI). We feel that the choice of title is misleading, as it does not specify the intervention. In addition, the authors also do not substantiate their claim that TFESI are in fact better than ILESI. In the abstract, it is noted that they included level one studies, but in the actual description of methods, under eligibility criteria, they state that they included level one and two studies. Since most readers access and read only the title and abstract [2], wrong interpretation about application of study findings could be drawn.

The authors had appropriately registered their trial at PROSPERO [3], a close look at the registered protocol and the published article reveals discrepancies. The outcome of recourse to surgery is not referred to in the final manuscript. They also state that only scores from similar periods post-injection will be pooled; however, they do not actually report this in their final manuscript. In fact, they report

Harsha Shanthanna

shanthh@mcmaster.ca

1 Department of Anaesthesia, St. Joseph's Healthcare, McMaster University, Hamilton, Canada

2 National Institute of Mental Health and Neurosciences, Bangalore, India diminution of pain scores at first visit as the outcome of interest for the statistical analysis in this paper. They excluded four RCTs (which were abstracts), as they could not assess their quality. However, the authors did not intend to exclude low-quality studies in their protocol. Had the results from the excluded RCTs been included, the effect size could have shifted to being significant on the side of particulate steroids.

Despite only including RCTs of lumbar TFESI, they had a large heterogeneity of $94 \%$, and significantly limited sample size overall. It is important to explain the reasons for such large heterogeneity, which we feel has not been attempted. In view of all these limitations, it is important that the findings of this systematic review need to be interpreted cautiously.

Compliance with ethical standards

Conflict of interest The authors declare that they have no conflict of interest.

\section{References}

1. Feeley IH, Healy EF, Noel J, Kiely PJ, Murphy TM (2016) Particulate and non-particulate steroids in spinal epidurals: a systematic review and meta-analysis. Eur Spine J. doi:10.1007/ s00586-016-4437-0

2. Barry HC, Ebell MH, Shaughnessy AF, Slawson DC, Nietzke F (2001) Family physicians' use of medical abstracts to guide decision making: style or substance? J Am Board Fam Pract 14:437-442

3. http://www.crd.york.ac.uk/PROSPERO/display_record.asp?ID= CRD42015025312. Accessed 14 March 2016 